\title{
The risk assessment of a crude oil pipeline using fuzzy and bayesian based bow-tie analysis
}

DOI:10.36909/jer.ICMMM.12399

\author{
Jishin Jayan T*, Muthukumar K*, V.R. Renjith**, Priscilla George**
}

*Department of Mechanical Engineering, Bannari Amman Institute of Technology, Sathyamangalam, Tamilnadu, India.

**Division of Safety and Fire Engineering, School of Engineering, Cochin University of Science and Technology (CUSAT), Kochi, Kerala, India.

*Email: jishin.jayan.jjt@gmail.com; Corresponding Author.

\begin{abstract}
Cross country pipelines are one of the major modes of transportation of petroleum products. Due to external and internal factors, the failure probability of pipeline is increasing over the past decades. The failure of pipelines can cause fires, which can result in environmental damage, loss of materials and further resulting into casualties. Effective risk assessment is vital to prevent and mitigate such potential incidents. In this work, an efficient tool that makes use of Bayesian approach along with Bow-tie analysis is used to obtain the failure frequencies of all the identified causes that may lead to failure of the selected pipeline located in Kerala, India. Bayesian networks can perform effective dynamic risk analysis by considering the conditional dependencies between various basic events leading to pipeline failure. The fuzzy logic and expert elicitation method are incorporated to determine the prior failure frequencies of all the identified causes of pipeline failure. The effectiveness of Bayesian network in performing forward analysis to determine the probabilities of pipeline failure consequences is demonstrated. This study also identifies the weak links associated with the occurrence of particular consequences, so that adequate measures can be taken to rectify them.
\end{abstract}

Key words: Risk analysis; Bow-tie analysis; Bayesian network; Event tree analysis; Fault tree analysis.

\section{INTRODUCTION}

Cross Country pipelines are one of the most convenient, economical and reliable method for transporting petroleum products. The intrinsic properties of petroleum products and their increasing industrial or commercial importance have led to a greater shift of petroleum products through pipelines. The failure of petroleum pipeline can occur due to several different causes such as internal and external corrosion, natural disaster, construction or material defects and external or third party interference. Therefore, an effective risk assessment is vital for 
preventing and mitigating possible accidents resulting from pipeline failures.

The bowtie analysis is one of the many quantitative risk management tools used in oil refineries, process plants, and various other sectors. The quantification of Bow-tie analysis requires the failure frequency of the basic events which are generally obtained from standard failure data sources. Bow-tie analysis is not capable of performing dynamic risk analysis due to its static nature and also has the disadvantage that they do not consider the conditional dependencies between different basic events leading to top event (Khakzad et al., 2012). Bayesian networks can overcome this limitation.

Bayesian networks are graphical representations of variables making use of nodes and associated conditional probability tables, the relation between the nodes are depicted through arcs (Yuan et al., 2015). The main benefit of using the Bayesian network is its likelihood updating feature due to its dynamic nature. The prior frequency values of the events or nodes are updated with the help of Bayes theorem to give the posterior probability values when new observations are made on the events or variables (Villa et al., 2016).

The objective of this study was to perform the risk analysis of a crude oil pipeline located in Kerala, India using Fuzzy based Bayesian approach and Bow-tie approach. Since the failure data sources for pipeline failures relevant to Indian conditions are sparse, both fuzzy logic and expert elicitation methods are used to obtain the failure probability values of basic events (Zarei et al., 2019; Yazdi and Kabir, 2017). The expert opinions expressed in linguistic terms are changed into failure probabilities by making use of fuzzy logic method (Renjith et al., 2010). Bayesian approach is used to identify the weak links associated with different consequences of pipeline failure by performing backward analysis.

\section{RISK ASSESSMENT TOOLS}

Risk assessment in industries is becoming increasingly important due to the occurrence of certain events that may affect the industries. There are several risk assessment tools in which the bow tie method is increasingly popular because it takes the advantages from the fault tree method as well as event tree method and can be utilized effectively to calculate the risk. However, the bow tie model quantification requires reliable probability failure data. The study by Jacinto and Silva (2010) shows that the quantification of bow-tie model is difficult because it requires the failure frequency values of all the events and the safety barriers which can be difficult to obtain when the model is used in complex environments.

In some literature, the Fault tree analysis is coupled with Fuzzy logic method and expert elicitation to overcome the problem of uncertainties within the input parameter( failure probability data) so to perform an effective risk analysis within the oil and gas 
industry(Cheliyan and Bhattacharyya, 2017).

The Bow-Tie model developed earlier may need to be updated based on new data, which is difficult due to the static nature of both fault tree and event tree. If new data are available within an industry, they should be used accurately to reflect the new state of the industry (Paltrinieri et al., 2014). In a study by Ferdous et al.,(2012) overcome the static nature problem by updating the initial Bow-tie with the integration of fuzzy and Bayesian approaches.

One of the main issues in the bow-tie analysis is the reliability of data cannot be assured when they are utilized in complex conditions. If the failure data is not reliable and inputting this values into the bow-tie elements will results into inefficient bow-tie analysis. The fuzzy logic method is one of the popular approach to address this absence of clear data (de Ruijter \& Guldenmund, 2015; Ferdous et al., 2012).

The Bayesian network is a probabilistic approach of inference, which can overcome the static constraint of the bow-tie method because of its probability update mechanism; it helps in effective implementation of linear prediction and diagnostic technique.

The backward diagnosis and forward prediction techniques can be carried out by using the principles of Bayes theorem as discussed below (Shan et al., 2017).

1) In forward prediction technique, the failure probability of the end state consequences is estimated based on the frequency rates of the basic events. Let the prior failure frequency of the basic event $\mathrm{X}_{i}$ be $P\left(\mathrm{X}_{i}\right)$, and the probability of the end state consequence or outcome event $\mathrm{C}$ is $P(\mathrm{C})$ and then conditional probability of outcome event $\mathrm{C}$ in the condition of the basic event $\mathrm{X} i$ be $P(\mathrm{C} \mid \mathrm{X} i)$, then according to the forward prediction technique $P(\mathrm{C})$ is given by,

$$
P(C)=\sum_{i=1}^{n} P\left(X_{i}\right)\left(C \mid X_{i}\right)
$$

2) In backward diagnosis technique, a consequence event is considered to have occurred and based on that the basic events that lead to certain consequence are diagnosed. Let the prior frequency value of the basic event $\mathrm{X} i$ be $P(\mathrm{X} i)$, and the conditional probability of the basic event $\mathrm{X} i$ in the condition of the consequence event $\mathrm{C}$ be $P(\mathrm{X} i \mid \mathrm{C})$; then according to the backward diagnosis technique $P(\mathrm{X} i \mid \mathrm{C})$ is given by,

$$
P\left(X_{i} \mid C\right)=\frac{P\left(C \mid \mathrm{X}_{i}\right) P(X)}{\sum_{i=1}^{n} P\left(C \mid \mathrm{X}_{i}\right) \mathrm{P}\left(\mathrm{X}_{i}\right)}
$$




\section{FRAMEWORK OF RISK ANAYSIS}

A generic framework of Bowtie analysis given by Ferdous et al.,(2013) is updated with the addition of Bayesian approach to determine the updated prior probability values or posterior values of the basic events based on evidence or new information. In the framework shown in Figure 1, the steps involved in the construction of fault tree section and event tree are represented along the either sides of the bow-tie diagram.

For the quantification of the bow-tie developed, we need reliable failure probability data relevant to the specific scenario which may not be available if bow-tie is used in the complex environment. In order to overcome the vagueness associated with these data, both fuzzy logic and expert elicitation methods are employed to quantify the failure frequency values of all basic events and safety barriers which are important for the quantification of the bow-tie. The bow-tie is charted into a Bayesian network which is more flexible and dynamic in nature as it allows for addition of new information as it becomes available.

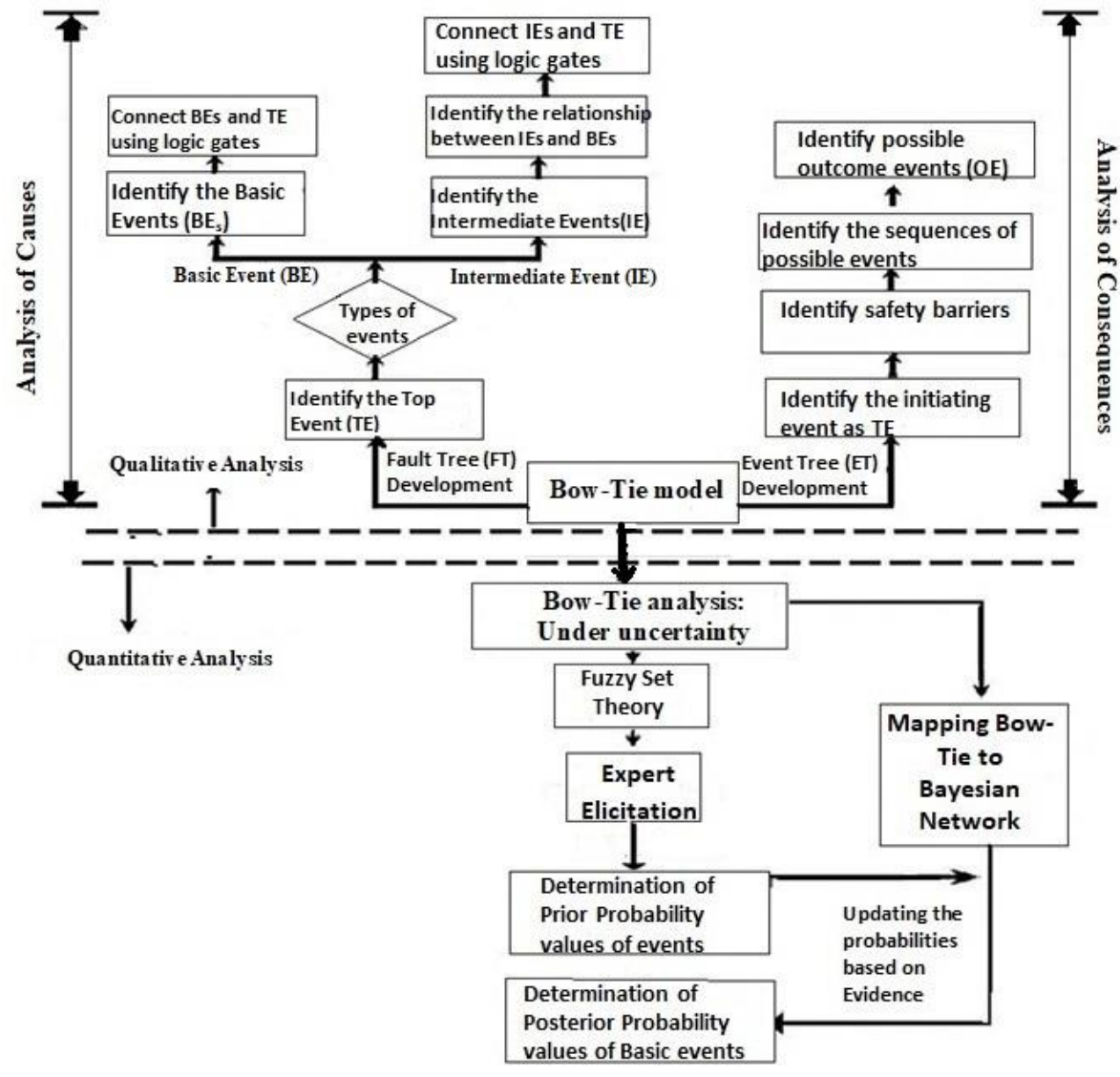

Figure 1 Framework of Risk analysis

\section{Estimation of failure probabilities}

The failure frequency data of all the identified basic events must be known in prior to determine the failure frequency of the undesirable event. This work incorporates both the Fuzzy logic method and expert judgment method to determine the failure frequencies of all the basic 
events. Experts from concerned field are selected and they were asked to provide judgments about the failure frequencies of the identified events based on their work experience.

Experts may not be able to accurately assess the failure frequencies of events, so linguistic terms like low, medium, high are used to describe the failure probabilities of events. In this study conversion scale 6 based on Saaty's scale (Saaty \& Ozdemir, 2003) is adopted to alter 5- point linguistic expressions to fuzzy number as shown in Table 1.

Table 1 Linguistic term to Fuzzy set conversion scale

\begin{tabular}{|c|c|}
\hline Linguistic terms & Fuzzy sets \\
\hline Very low & $(0,0,0.1,0.2)$ \\
\hline Low & $(0.1,0.25,0.25,0.4)$ \\
\hline Medium & $(0.3,0.5,0.5,0.7)$ \\
\hline High & $(0.6,0.75,0.75,0.9)$ \\
\hline Very high & $(0.8,0.9,1,1)$ \\
\hline
\end{tabular}

Similarity Aggregation Method is used in the present study for aggregating experts' judgment to a single fuzzy number set. Similarity Aggregation Method is a simple method which includes fetching together the judgements from experts, for a certain basic event (Shan et al., 2017; Lavasani et al., 2015).Steps of Similarity Aggregation Method are described as follows:-

1. Estimation of Degree of Agreement amongst Expert $p$ and Expert $q$.

Consider $E p=(a 1, a 2, a 3, a 4)$ and $E q=(b 1, b 2, b 3, b 4)$ are standard trapezoidal fuzzy numbers. The total number of experts' is represented by $M$. The agreement degree of expert $p$ and expert $q$ is given as

$$
S_{p q}=1-\frac{1}{4} \sum_{i=1}^{4}\left|a_{i}-b_{i}\right|
$$

2. Estimation of Experts' Average Agreement (A) Degree, is calculated by given equation.

$$
A_{p}=\frac{1}{M-1} \sum_{n=1}^{M} S_{p q}
$$

3. Estimation of Experts' Relative Agreement (R) Degree., is calculated by given equation $\mathrm{R}_{\mathrm{p}}$.

$$
R_{p}=\frac{A_{p}}{\sum_{K=1}^{M} A_{K}}
$$

4. Estimation of Experts' Consensus Coefficient (C) Degree., is calculated by given equation $\mathrm{C}_{\mathrm{p}}$.

$$
C_{P}=\beta \cdot W_{p}+(1-\beta) R_{p}
$$

Where' $\beta$ ' is a factor known as a relaxation factor

The relaxation factor is usually given by the decision maker based on the quality of 
judgment obtained from each expert; $\beta \in[0,1]$.

' $w_{p}$ ' is the weighting factor of the expert which is estimated as shown in table 4.

5. Estimation of Aggregated Result of Experts' judgments. This is given by the equation $\mathrm{E}_{\mathrm{AG}}$.

$$
E_{A G}=C_{1} \times E_{1}+C_{2} \times E_{2}+\ldots . .+C_{M} \times E_{M}
$$

The center area defuzzification method is introduced to convert the trapezoidal fuzzy set $\mathrm{E}_{\mathrm{AG}}=(\mathrm{n} 1, \mathrm{n} 2, \mathrm{n} 3, \mathrm{n} 4)$ to a crisp number.

$$
\begin{gathered}
X^{*}=\frac{\int_{n_{1}}^{n_{2}}\left\{\left(\frac{x-n_{1}}{n_{2}-n_{1}}\right) x d x+\int_{n_{2}}^{n_{3}} x d x+\int_{n_{3}}^{n_{4}}\left(\frac{n_{4}-x}{n_{4}-n_{3}}\right)\right\} x d x}{\int_{n_{1}}^{n_{2}}\left\{\left(\frac{x-n_{1}}{n_{2}-n_{1}}\right) d x+\int_{n_{2}}^{n_{3}} d x+\int_{n_{3}}^{n_{4}}\left(\frac{n_{4}-x}{n_{4}-n_{3}}\right)\right\} d x} \\
=\frac{1}{3} \frac{\left(n_{4}+n_{3}\right)^{2}-n_{4} n_{3}-\left(n_{1}+n_{2}\right)^{2}+n_{1} n_{2}}{\left(n_{4}+n_{3}-n_{1}-n_{2}\right)}
\end{gathered}
$$

Finally, the failure probability value' $P r$ ' can be calculated using the equation proposed by Onisawa,(1988) which can convert crisp number obtained after defuzzification in to failure probability

$$
\operatorname{Pr}=\left\{\begin{array}{cc}
\frac{1}{10^{k}}, & X^{*} \neq 0 \\
0, & X^{*}=0
\end{array} \quad \text { where, } K=2.301 \times\left[\frac{1-X^{*}}{X^{*}}\right]^{\frac{1}{3}}\right.
$$

\section{RISK ANALYSIS STUDY ON A PIPELINE: - A CASE STUDY}

The risk analysis is carried out on a cross-country crude oil pipeline section of 30" API5L Gr. X52 of thickness $10.3 \mathrm{~mm} / 14.3 \mathrm{~mm}$ and $20 \mathrm{~km}$ long. This pipeline is used for carrying the crude oil from Shore Tank Farm (STF) located at Puthuvypeen to Kochi Refinery, Kerala.

\section{Fault tree construction of pipeline Failure}

The fault tree shows possible factors that can cause failure of the pipeline, the fault trees are usually placed on the left side of the bowtie model. In this study, the failure of the crude oil pipeline is taken as the critical event or top event in the constructed fault tree. To assess the risk of pipeline failure, factors that could cause pipeline failure must be analyzed in the beginning. The fault tree is constructed based on identified causes that lead to the pipeline failure which can be obtained from the past history of the pipeline failure data or through the comprehensive analysis of pipelines design standards, operation, construction and maintenance. The factors that lead to pipeline failure are categorized as external factors and internal factors. The external factors comprises of corrosion, third party interference, natural disasters, incorrect operations and maintenance on the pipeline. The internal factors that lead to the pipeline failure are 
construction defects such as improper way of laying the pipelines and improper welding between the pipeline materials, and material defects such as the presence of inclusions and cracks within the material.

The fault tree for the crude oil pipeline under study was developed with the pipeline failure as the top event (left hand side of Figure 2). This fault tree contains of 14 basic events whose descriptions are given in Table 2. The top event or critical event, intermediate event and basic events are connected using the logic gates. The top event failure frequency can be determined easily by solving the Boolean gates in the fault tree.

Table 2 Identified basic events and their descriptions

\begin{tabular}{|c|c|c|c|}
\hline \multicolumn{2}{|c|}{ Intermediate Events } & Basic Events & Description \\
\hline \multirow{10}{*}{$\begin{array}{l}\text { External } \\
\text { Factors }\end{array}$} & \multirow{4}{*}{ Corrosion } & $\mathrm{B} 1$ & $\begin{array}{l}\text { Failure of pipeline coating may leads to } \\
\text { external corrosion }\end{array}$ \\
\hline & & $\mathrm{B} 2$ & $\begin{array}{l}\text { Failure of cathodic protection to the } \\
\text { pipeline may leads to external corrosion }\end{array}$ \\
\hline & & B3 & $\begin{array}{l}\text { Failure to use inhibitors may result in } \\
\text { internal corrosion of the pipeline }\end{array}$ \\
\hline & & B4 & $\begin{array}{l}\text { The presence of water or acid mediums } \\
\text { can cause internal corrosion }\end{array}$ \\
\hline & \multirow{2}{*}{$\begin{array}{l}\text { Interference } \\
\text { from Third } \\
\text { party }\end{array}$} & B5 & $\begin{array}{l}\text { Third party interference due to parties } \\
\text { ignore signage }\end{array}$ \\
\hline & & B6 & $\begin{array}{l}\text { Third party interference party due to } \\
\text { sabotage }\end{array}$ \\
\hline & \multirow{2}{*}{$\begin{array}{l}\text { Natural } \\
\text { disaster }\end{array}$} & B7 & Flood (natural disaster) \\
\hline & & B8 & Earth quake (natural disaster) \\
\hline & \multirow{2}{*}{$\begin{array}{l}\text { Incorrect } \\
\text { operations or } \\
\text { maintenance }\end{array}$} & B9 & $\begin{array}{l}\text { Risk of operational error due to } \\
\text { incorrect operations }\end{array}$ \\
\hline & & B10 & $\begin{array}{l}\text { Risk maintenance error due poor } \\
\text { maintenance of pipeline and its } \\
\text { auxiliaries }\end{array}$ \\
\hline \multirow{4}{*}{$\begin{array}{l}\text { Internal } \\
\text { Factors }\end{array}$} & \multirow{2}{*}{$\begin{array}{l}\text { Material } \\
\text { defect }\end{array}$} & B11 & $\begin{array}{l}\text { Risk of failure of material due to } \\
\text { improper selection of pipeline material }\end{array}$ \\
\hline & & B12 & $\begin{array}{l}\text { Risk of material defect due to presence } \\
\text { of cracks and inclusions }\end{array}$ \\
\hline & \multirow{2}{*}{$\begin{array}{l}\text { Construction } \\
\text { defect }\end{array}$} & B13 & $\begin{array}{l}\text { Risk of construction defect due to } \\
\text { failure of welded joints }\end{array}$ \\
\hline & & B14 & $\begin{array}{l}\text { Risk of construction defect due to } \\
\text { improper way of laying }\end{array}$ \\
\hline
\end{tabular}

\section{Event tree analysis}

The event tree analysis is a risk analysis technique, which discovers various outcomes from an initiating event depending upon the success and failure of escalation factors or barriers and establishes a path which helps to evaluate the occurrence probabilities of end state outcomes. The oil and gas pipeline failure can result in potential consequences such as deflagration, 
confined vapour cloud explosion, jet fire, flash fire, and material loss which may further result in environment damages and casualties. The barriers such as immediate ignition, delayed ignition and the existence of space confinement are considered to have various impacts on pipeline failure to have different consequences. Event Tree proposed by Shahriar et al.,(2012) has been adopted in this study. In the event tree, the failure of the pipeline is selected as the initiating or originating event and based on the existence of different barriers all the possible consequences of the accident are obtained (right hand side of Figure 2).

Once the failure probabilities of the initiating event and escalation factors or safety barriers are determined then the occurrence probability values of the end state consequences can be easily identified by the quantification of the event tree. For example, the occurrence probability value of the second consequence (jet fire) $\mathrm{C} 2$ in Figure 2 can be calculated as:

$$
\mathrm{P}(\mathrm{C} 2)=\mathrm{P}(\mathrm{T}) \mathrm{P}(\mathrm{S} 1) \mathrm{P}(\overline{\mathrm{S} 3})
$$

Where $\mathrm{P}(\mathrm{T})$ is the probability value of the critical event, and $\mathrm{P}(\mathrm{S} 1), \mathrm{P}(\mathrm{S} 2)$ and $\mathrm{P}(\mathrm{S} 3)$ refer to the probability of S1(immediate ignition),S2(delayed ignition),S3(space confinement) occurring and $\mathrm{P}(\overline{\mathrm{S} 3})$ represents probability of not occurring the event $\mathrm{S} 3$.

\section{Bow-tie model construction}

In Bow-tie generally, the fault tree analysis categorizes the cause events that can lead to an undesired critical event, while the Event tree analysis identifies the sequences of events from the initiating event to the different accident scenarios. The bow-tie diagram of the failure of the crude oil pipeline is shown in Figure 2.

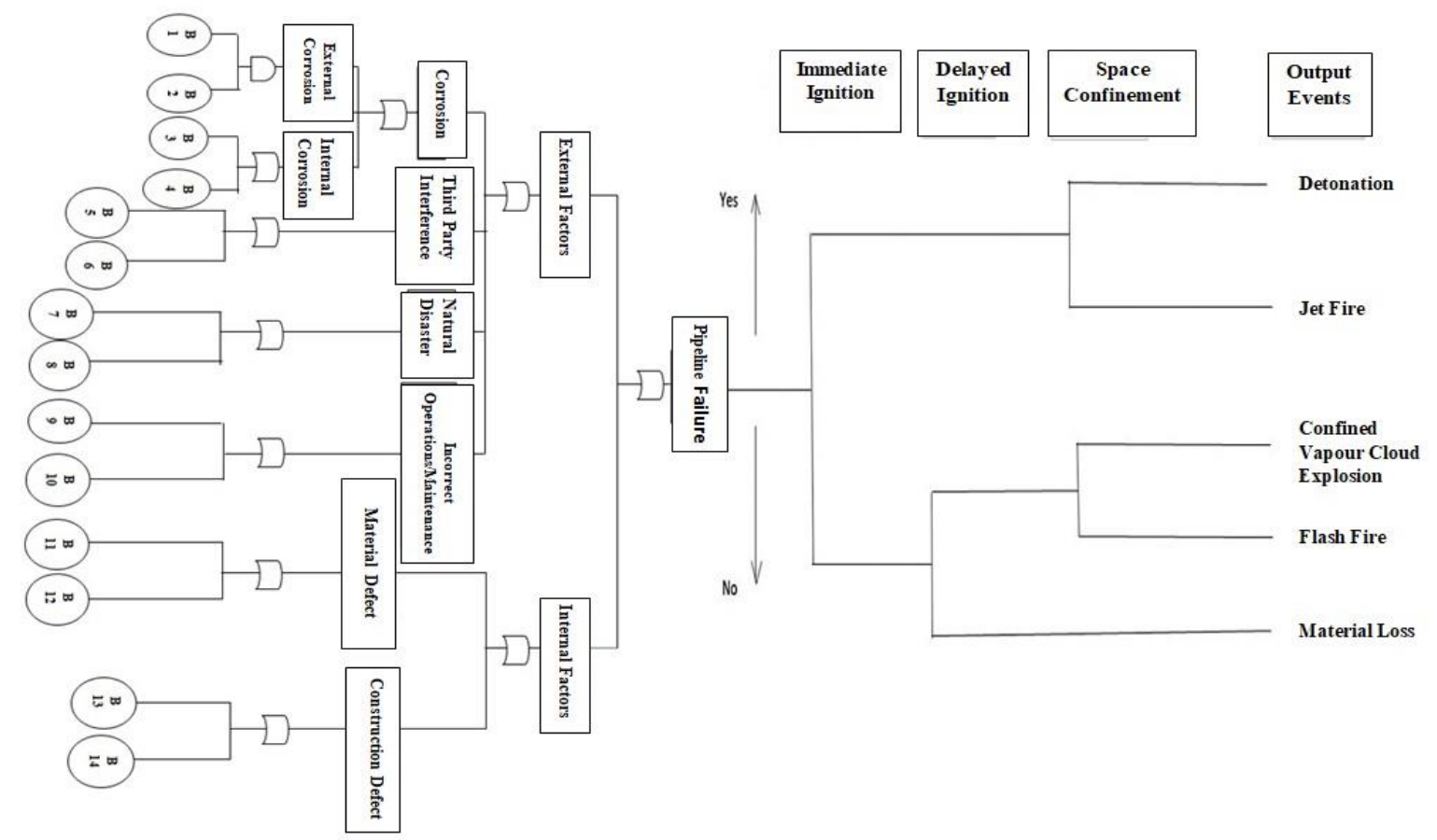

Figure 2 Bow-Tie model of crude oil pipeline failure 


\section{Basic event failure probability calculation}

To calculate the basic event failure probabilities by expert elicitation method, three experts from concerned field of the crude oil pipeline are selected for obtaining their judgments on the various factors affecting the pipeline. The expert's organizational position, level of education, and service experience are considered in the selection process of experts, it is carried out mainly to know the level of value associated with each expert judgment. The experts weight factor is presented to reflect the relative worthiness of the selected professionals (Table 3 and Table 4).The experts give their opinion in linguistic expressions which are transformed to fuzzy number using conversion scale 6 and these fuzzy numbers are then aggregated using similarity aggregation method where the experts weighing scores based on their experience and qualifications are also considered. The aggregated fuzzy number is then defuzzified into a distinct crisp value using the center area method of defuzzification and finally, the basic event failure frequencies are calculated. The 14 basic events failure frequency values and failure frequencies of the safety barriers are calculated based on fuzzy logic and expert elicitation logic as mentioned in previous section and shown in Table 5.

Table 3 Weighting score and classification of different experts

\begin{tabular}{|c|c|c|}
\hline Constitution & $\begin{aligned} \text { Classifications } \\
\end{aligned}$ & Score \\
\hline \multirow{5}{*}{ Organizational Positions } & Senior manager & 5 \\
\hline & Manager & 4 \\
\hline & Assistant Manager & 3 \\
\hline & supervisors & 2 \\
\hline & Workers & 1 \\
\hline \multirow{5}{*}{ Service Experience } & $\geq 30$ years & 5 \\
\hline & $20-29$ years & 4 \\
\hline & $10-19$ years & 3 \\
\hline & $5-9$ years & 2 \\
\hline & $\leq 5$ years & 1 \\
\hline \multirow{5}{*}{ Educational qualification } & $\mathrm{PhD}$ & 5 \\
\hline & Master & 4 \\
\hline & Bachelor & 3 \\
\hline & Diploma & 2 \\
\hline & School Level & 1 \\
\hline
\end{tabular}


Table 4 Experts Weighting Score and Weighting factor

\begin{tabular}{|l|l|l|l|l|l|}
\hline Experts & $\begin{array}{l}\text { Professional } \\
\text { Positions }\end{array}$ & $\begin{array}{l}\text { Service } \\
\text { Experience }\end{array}$ & $\begin{array}{l}\text { Educational } \\
\text { qualification }\end{array}$ & $\begin{array}{l}\text { Weighting } \\
\text { score }\end{array}$ & $\begin{array}{l}\text { Weighting } \\
\text { factor }\end{array}$ \\
\hline EXPERT 1 & Chief Manger & 18 Years & $\begin{array}{l}\text { Bachelor } \\
\text { Degree }\end{array}$ & $5+3+3=11$ & 0.392 \\
\hline EXPERT 2 & Manager & 11 Years & $\begin{array}{l}\text { Bachelor } \\
\text { Degree }\end{array}$ & $4+3+3=10$ & 0.357 \\
\hline EXPERT 3 & $\begin{array}{l}\text { Assistant } \\
\text { Manager }\end{array}$ & 3 Years & $\begin{array}{l}\text { Bachelor } \\
\text { Degree }\end{array}$ & $3+1+3=7$ & 0.25 \\
\hline
\end{tabular}

For expert 'i', weighting score and the weighting factor is calculated as follows:

Weighting score $\left(W_{i}\right)=$ Organizational Position score + service time score +

Educational level score

Let ' $M$ ' be the number of experts. Weighting Factor for expert ' $i$ ' is given by,

$$
E_{i}=\frac{W_{i}}{\sum_{i=1}^{M} W_{i}}
$$

Table 5 Probability values of Basic events and Safety Barriers

\begin{tabular}{|c|c|c|c|c|c|c|c|}
\hline Basic & Linguistic & Agg & sated ex & eert juc & sement & Crisp & Prior \\
\hline B1 & $\begin{array}{l}\text { Low, Medium, } \\
\text { Medium }\end{array}$ & 0.221 & 0.401 & 0.401 & 0.581 & 0.46 & $3.74 \times 10^{-3}$ \\
\hline B2 & $\begin{array}{l}\text { Medium, Medium, } \\
\text { Low }\end{array}$ & 0.249 & 0.436 & 0.436 & 0.624 & 0.437 & $3.136 \times 10^{-3}$ \\
\hline B3 & Low, Low, Low & 0.099 & 0.248 & 0.248 & 0.3977 & 0.247 & $4.61 \times 10^{-4}$ \\
\hline B4 & $\begin{array}{l}\text { Low, Low, } \\
\text { Medium }\end{array}$ & 0.194 & 0.389 & 0.389 & 0.584 & 0.389 & $2.114 \times 10^{-3}$ \\
\hline B5 & $\begin{array}{l}\text { Very Low, Low, } \\
\text { Low }\end{array}$ & 0.078 & 0.195 & 0.234 & 0.390 & 0.204 & $2.38 \times 10^{-4}$ \\
\hline B6 & $\begin{array}{l}\text { Very Low, Very } \\
\text { Low, Very Low }\end{array}$ & 0 & 0 & 0.099 & 0.198 & 0.077 & $5.41 \times 10^{-6}$ \\
\hline B7 & $\begin{array}{l}\text { Very Low, Very } \\
\text { Low, Very Low }\end{array}$ & 0 & 0 & 0.099 & 0.198 & 0.077 & $5.41 \times 10^{-6}$ \\
\hline B8 & $\begin{array}{l}\text { Very Low, Low, } \\
\text { Very Low }\end{array}$ & 0.035 & 0.087 & 0.151 & 0.268 & 0.138 & $5.78 \times 10^{-5}$ \\
\hline B9 & $\begin{array}{l}\text { Very Low, Low, } \\
\text { Low }\end{array}$ & 0.078 & 0.195 & 0.234 & 0.390 & 0.204 & $2.38 \times 10^{-4}$ \\
\hline B10 & $\begin{array}{l}\text { Very Low, Very } \\
\text { Low, Very Low }\end{array}$ & 0 & 0 & 0.099 & 0.198 & 0.077 & $5.41 \times 10^{-6}$ \\
\hline B11 & $\begin{array}{l}\text { Very Low, Very } \\
\text { Low, Very Low }\end{array}$ & 0 & 0 & 0.099 & 0.198 & 0.077 & $5.41 \times 10^{-6}$ \\
\hline B12 & $\begin{array}{l}\text { Very Low, Very } \\
\text { Low, Very Low }\end{array}$ & 0 & 0 & 0.099 & 0.198 & 0.077 & $5.41 \times 10^{-6}$ \\
\hline B13 & $\begin{array}{l}\text { Very Low, Very } \\
\text { Low, Very Low }\end{array}$ & 0 & 0 & 0.099 & 0.198 & 0.077 & $5.41 \times 10^{-6}$ \\
\hline
\end{tabular}




\begin{tabular}{|l|l|l|l|l|l|l|l|}
\hline B14 & $\begin{array}{l}\text { Very Low, Very } \\
\text { Low, Very Low }\end{array}$ & 0 & 0 & 0.099 & 0.198 & 0.077 & $5.41 \times 10^{-6}$ \\
\hline S1 & Low, Low, Low & 0.099 & 0.248 & 0.248 & 0.397 & 0.247 & $4.61 \times 10^{-4}$ \\
\hline S2 & Low, Medium,Low & 0.169 & 0.335 & 0.335 & 0.502 & 0.334 & $1.27 \times 10^{-3}$ \\
\hline S3 & $\begin{array}{l}\text { Medium, Medium, } \\
\text { Low }\end{array}$ & 0.249 & 0.436 & 0.436 & 0.624 & 0.437 & $3.136 \times 10^{-3}$ \\
\hline S4 & Low, Low, Low & 0.099 & 0.248 & 0.248 & 0.397 & 0.247 & $4.61 \times 10^{-4}$ \\
\hline
\end{tabular}

\section{Bayesian network for the pipeline failure}

Bayesian network is modeled by mapping the bow-tie model; the mapping method in Khakzad et al., (2013) is taken in this study. The network shows the relationship of all the identified basic events (root nodes), top event (pivot node) and the consequences through the links or arcs as shown in Figure 3. The network is constructed from the bow-tie model developed, where each event from the bow-tie is converted into nodes and each node is connected logically through arcs. For each root node the failure probability values obtained from the quantified bow-tie is assigned and for the other nodes, conditional probability tables are provided based on the logic gates.

The Bayesian network can be analysed through forward prediction and backward diagnosis analysis. The forward prediction technique in the Bayesian is used to determine the occurrence frequency of end state consequences from the obtained failure frequency values of the cause events and escalation factors or barriers. The estimation of occurrence frequency values of outcome events can be carried out easily by quantifying the bow-tie model. The occurrence frequency values of each consequence events obtained from the forward Bayesian technique and from the bow-tie model is found to be same.

Bayesian Network takes benefit of Bayes theorem which helps to update the prior frequency data of events based on the given new information known as evidence, thus resulting into an updated prior data (posterior data). The effect of the evidence can be transmitted through the Bayesian network, updating the probability distribution of the nodes that are related to the new information or evidence. The backward linear prediction diagnostic analysis is carried out to update the frequency prior data of basic events based on evidence. Figure 3 shows the backward analysis performed using GeNIe software (version 2.3, Academic edition), where the evidence is set as occurrence of jet fire.

The failure of the pipeline and the consequence the jet fire are taken as evidence to find the 
updated frequency values of the cause events based on the evidence provided.The prior and posterior probability values for all basic events are listed in Table 7 and it can be observed that the updated probability values are greater than the prior probability values for most basic events. By analysing posterior data based on the evidence (Jet fire and pipeline failure) the most likely causes of the jet fire are internal corrosion and the interference from the third party, which are having higher posterior values and these factors are considered to be the weak link in the network through which there is a higher chance of accident evolution.

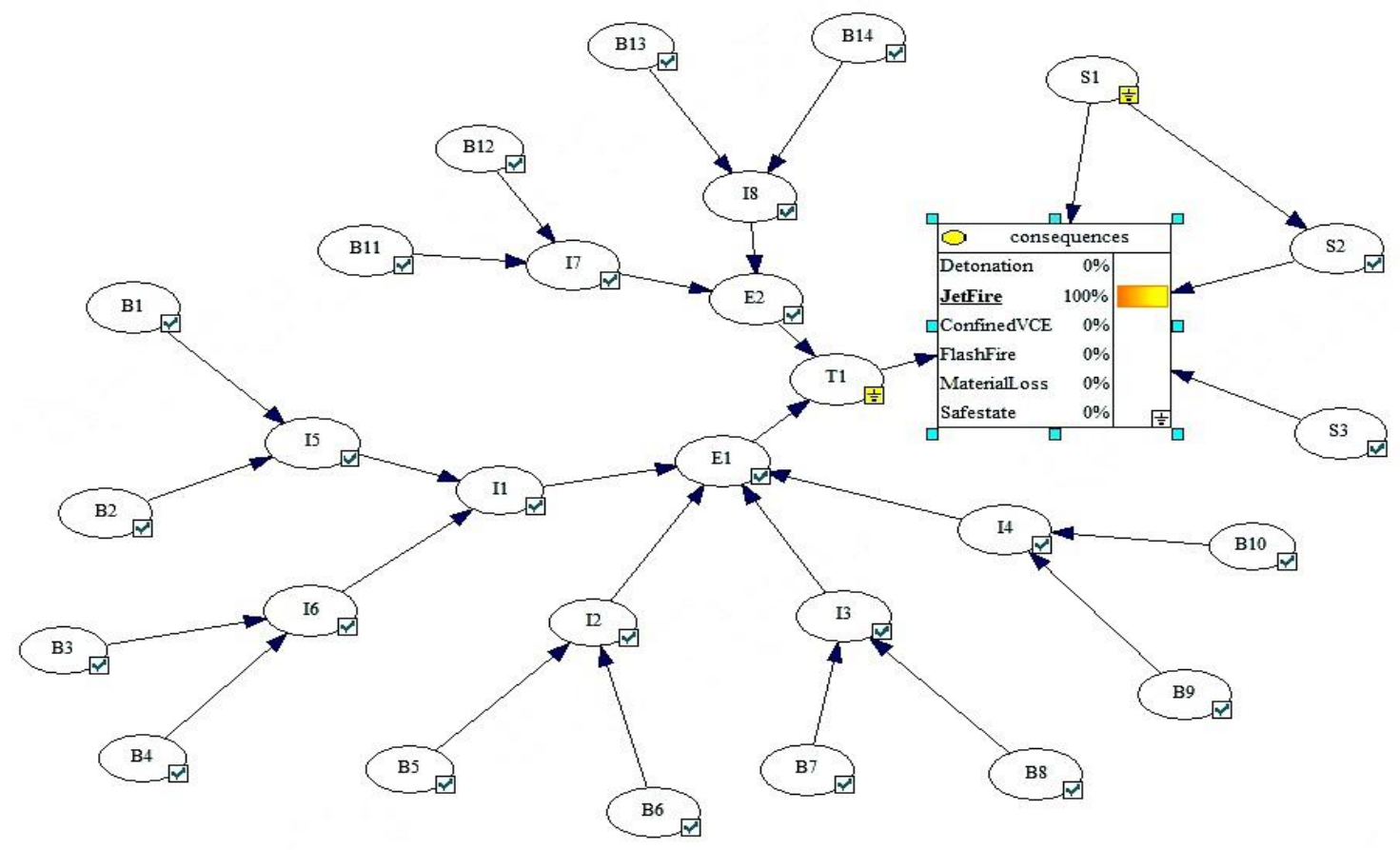

Figure 3 Bayesian network (Backward diagnostic analysis)

B1 - B14 represents basic events from Bow tie;

I1 - I8, E1and E2 Represents Intermediate events from Bow tie;

T1 - Represents the top event (Pipeline failure)

S1, S2, S3 - Represents barriers that could leads to different possible outcomes

\section{RESULTS AND DISCUSSIONS}

- A Bow Tie diagram is framed based upon the identified causes and consequences (See Figure 2).

- The Probability data of all basic events are obtained and are tabulated (see Table 5) using Fuzzy logic and Expert elicitation.

- The Probability value of Pipeline Failure (Top event) is estimated by solving the gates of fault tree constructed and value obtained is $3.14 \times 10^{-3}$ 
- The outcome events occurrence probability values are obtained by quantifying the event tree and the results obtained are tabulated as follows-

Table 6 Occurrence probability values of Consequence outcomes

\begin{tabular}{|c|c|c|}
\hline Consequence outcomes & Description & Occurrence probability values \\
\hline C1 & Detonation & $4.532 \times 10^{-9}$ \\
\hline C2 & Jet Fire & $1.442 \times 10^{-6}$ \\
\hline C3 & $\begin{array}{c}\text { Confined Vapor Cloud } \\
\text { Explosion }\end{array}$ & $1.247 \times 10^{-8}$ \\
\hline C4 & Flash Fire & $3.969 \times 10^{-6}$ \\
\hline C5 & Material Loss & $3.13 \times 10^{-3}$ \\
\hline
\end{tabular}

- Bayesian network is developed from the Bowtie by mapping all the events to nodes. From the Bayesian network constructed, the consequence Jet fire is taken as the evidence or information and the failure frequency values of all basic events are simplified based on this evidence, and the results obtained are tabulated as follows-

Table 7 Basic events failure probability values

\begin{tabular}{|c|c|c|}
\hline Basic events & Prior probability values & $\begin{array}{c}\text { Posterior probability } \\
\text { values }\end{array}$ \\
\hline B1 & $3.74 \times 10^{-3}$ & $7.44 \times 10^{-3}$ \\
\hline B2 & $3.136 \times 10^{-3}$ & $6.84 \times 10^{-3}$ \\
\hline B3 & $4.61 \times 10^{-4}$ & $1.47 \times 10^{-1}$ \\
\hline B4 & $2.114 \times 10^{-3}$ & $6.74 \times 10^{-1}$ \\
\hline B5 & $2.38 \times 10^{-4}$ & $7.59 \times 10^{-2}$ \\
\hline B6 & $5.41 \times 10^{-6}$ & $1.72 \times 10^{-3}$ \\
\hline B7 & $5.41 \times 10^{-6}$ & $1.72 \times 10^{-3}$ \\
\hline B8 & $5.78 \times 10^{-5}$ & $1.84 \times 10^{-2}$ \\
\hline B9 & $2.38 \times 10^{-4}$ & $7.59 \times 10^{-2}$ \\
\hline B10 & $5.41 \times 10^{-6}$ & $1.72 \times 10^{-3}$ \\
\hline B11 & $5.41 \times 10^{-6}$ & $1.73 \times 10^{-5}$ \\
\hline B12 & $5.41 \times 10^{-6}$ & $1.73 \times 10^{-5}$ \\
\hline B13 & $5.41 \times 10^{-6}$ & $1.73 \times 10^{-5}$ \\
\hline B14 & $5.41 \times 10^{-6}$ & $1.73 \times 10^{-5}$ \\
\hline
\end{tabular}




\section{CONCLUSIONS}

In this study, a risk analysis of a cross country pipeline located in India is carried out using Bow-tie model coupled with Fuzzy and Bayesian approaches. The probability value of pipeline failure (Top event) is estimated as $3.14 \times 10^{-3}$ from the fault tree and validated by forward prediction of Bayesian network. The obtained failure probability value implies that there is only a less chance of causing the failure of the crude pipeline. By comparing all the factors affecting the pipeline failure, the corrosion factor is found to be the leading cause that may lead to the failure of this particular pipeline.

The occurrence probability values of the end state outcomes or consequences obtained through quantifying the Bow-tie model and the probability values of consequences obtained through the forward prediction technique in the Bayesian network are the same. Due to the dynamicity of the components, bow-tie model cannot be used for backward diagnosis where a particular consequence is selected as an evidence to update the frequency data of all the causes or the basic events. Hence in this study the static bow-tie model is converted into a Bayesian network which is dynamic in nature and it can be used to update prior frequency data of the events based on an information or evidence.

The updated (posterior) probability values of basic events based on given evidence are obtained after carrying out the backward diagnosis technique from the network created, which is used to find the weakest link present in the Bayesian network of pipeline failure. From the Bayesian network constructed, the consequence Jet fire is taken as evidence or information and the failure frequency values of all basic events are updated based on this evidence. The most probable accident evolution paths based on the evidence is the risk of internal corrosion mainly due to failure to use inhibitors and the presence of water and acid mediums, third party interference and operational errors. It is important to strengthen these weak links in the network in order to prevent the happening of the accident. By analysing the obtained posterior values of the basic events, measures can be developed in the future to prevent the occurrence of this predicted accident.

The failure data taken for the study is primarily based on expert judgments. To improve the quality of this risk analysis study historical research and statistical data that are applicable to the area under study can be considered in future. The results obtained from this study can help authorities to develop further measures to mitigate such potential accidents and to decide where to take corrective and preventive actions to assist in the risk management process. 


\section{REFERENCES}

Cheliyan, A. S., \& Bhattacharyya, S. K. 2017. Fuzzy fault tree analysis of oil and gas leakage in subsea production systems. Journal of Ocean Engineering and Science, 3(1), 38-48.

de Ruijter, A., \& Guldenmund, F. 2015. The bowtie method: A review. Safety Science, 88, 211-218.

Ferdous, R., Khan, F., Sadiq, R., Amyotte, P., \& Veitch, B. 2012. Handling and updating uncertain information in bow-tie analysis. Journal of Loss Prevention in the Process Industries, 25(1), 8-19.

Ferdous, R., Khan, F., Sadiq, R., Amyotte, P., \& Veitch, B. 2013. Analyzing system safety and risks under uncertainty using a bow-tie diagram: An innovative approach. Process Safety and Environmental Protection, 91(1-2), 1-18.

Jacinto, C., \& Silva, C. 2010. A semi-quantitative assessment of occupational risks using bowtie representation. Safety Science, 48(8), 973-979.

Khakzad, N., Khan, F., \& Amyotte, P. 2012. Dynamic risk analysis using bow-tie approach. Reliability Engineering and System Safety.

Khakzad, N., Khan, F., \& Amyotte, P. 2013. Dynamic safety analysis of process systems by mapping bow-tie into Bayesian network. Process Safety and Environmental Protection, 91(1-2), $46-53$.

Lavasani, S. M., Ramzali, N., Sabzalipour, F., \& Akyuz, E. 2015. Utilisation of Fuzzy Fault Tree Analysis (FFTA) for quantified risk analysis of leakage in abandoned oil and natural-gas wells. Ocean Engineering, 108, 729-737.

Onisawa, T. 1988. An approach to human reliability in man-machine systems using error possibility. Fuzzy Sets and Systems, 27(2), 87-103.

Paltrinieri, N., Khan, F., Amyotte, P., \& Cozzani, V. 2014. Dynamic approach to risk management: Application to the Hoeganaes metal dust accidents. Process Safety and Environmental Protection, 92(6), 669-679.

Renjith, V. R., G.Madhu, Nayagam, V. L. G., \& Bhasi, A. B. 2010. Two-dimensional fuzzy fault tree analysis for chlorine release from a chlor-alkali industry using expert elicitation. Journal of Hazardous Materials, 183(1-3), 103-110.

Saaty, T. L., \& Ozdemir, M. S. 2003. Why the magic number seven plus or minus two. Mathematical and Computer Modelling.

Shahriar, A., Sadiq, R., \& Tesfamariam, S. 2012. Risk analysis for oil \& gas pipelines: A sustainability assessment approach using fuzzy based bow-tie analysis. Journal of Loss Prevention in the Process Industries, 25(3), 505-523. 
Shan, X., Liu, K., \& Sun, P. L. 2017. Risk Analysis on Leakage Failure of Natural Gas Pipelines by Fuzzy Bayesian Network with a Bow-Tie Model. Scientific Programming, 2017(2). Villa, V., Paltrinieri, N., Khan, F., \& Cozzani, V. 2016. Towards dynamic risk analysis: A review of the risk assessment approach and its limitations in the chemical process industry. Safety Science.

Yazdi, M., \& Kabir, S. 2017. A fuzzy Bayesian network approach for risk analysis in process industries. Process Safety and Environmental Protection.

Yuan, Z., Khakzad, N., Khan, F., \& Amyotte, P. 2015. Risk analysis of dust explosion scenarios using bayesian networks. Risk Analysis, 35(2), 278-291.

Zarei, E., Khakzad, N., Cozzani, V., \& Reniers, G. 2019. Safety analysis of process systems using Fuzzy Bayesian Network (FBN). Journal of Loss Prevention in the Process Industries. 